\title{
Das Gesundheitswesen zwischen Kosten und Nutzen*
}

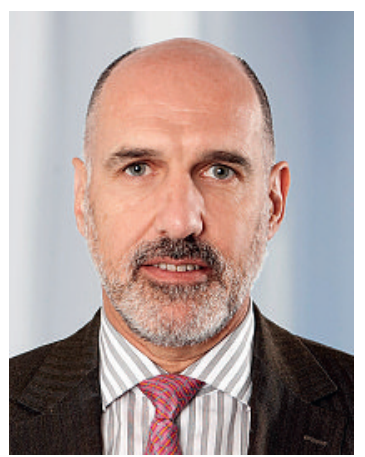

Wir Ärzte sind in einem Dilemma. Wir wurden in ein Spannungsfeld zwischen Kostendruck und Qualität der medizinischen Versorgung gedrängt. Auch wir wollen dazu beitragen, dass die Kosten für die medizinische Versorgung nicht explodieren, das haben wir in letzter Zeit überzeugend gezeigt.

Gleichzeitig wollen wir, dass die Patientinnen und Patienten möglichst rasch wieder gesund werden, oder, falls nicht möglich, so wollen wir ihnen ein weitgehend schmerzfreies und selbstbestimmtes Leben mit der Krankheit ermöglichen, damit sie weiterhin ihr ganzes Potenzial entfalten können.

Das kostet natürlich. Und doch, unser Anspruch ist legitim. Ethisch, für die Betroffenen und ihre Angehörigen, aber und vielleicht mehr als uns zurzeit bewusst ist - auch ökonomisch. Die Politik hingegen befasst sich seit gewisser Zeit fast ausschliesslich mit den Kosten.

\section{Wir sind in einem Spannungsfeld zwischen Kosten- druck und Qualität der medizinischen Versorgung}

Der TARMED wurde eingeführt, der Taktfahrplan der ärztlichen Leistungserbringung. Er gibt vor, wie viel Zeit für die Erbringung einer ärztlichen Leistung zur Verfügung stehen darf. Wird für die optimale Behandlung eines Patienten zusätzliche Zeit benötigt und diese abgerechnet, läuft der Arzt Gefahr, mit den Versicherern in Konflikt zu geraten. Beschlossen hat man die DRG - der Taktfahrplan für den Spitalaufenthalt. Er gibt vor, wie viel Zeit bei einer bestimmten Diagnose abgegolten werden kann. Benötigt der Heilungsprozess mehr Zeit, ohne dass klassifizierbare Komplikationen auftreten, so gerät das Spital in einen Interessenkonflikt.

Es muss gespart werden. Die Wirtschaftlichkeit muss gefördert werden. Die Effizienz muss gefördert werden. Doch nicht auf Kosten der Ärzte und schon gar nicht auf Kosten des Patientenwohles. Die Schmerzgrenze ist erreicht!

Fakt ist: Das Schweizer Gesundheitswesen ist hervorragend. Deshalb fühlen sich $86 \%$ der Schweizerinnen und Schweizer gesund. Die Kindersterblichkeit konnte in den letzten 30 Jahren um $45 \%$ gesenkt werden, im Gegenzug ist die Lebenserwartung um über 5 Jahre gestiegen. Aber vor allem haben wir es geschafft, frühzeitige Todesfälle zu minimieren und den Tod an den Lebensabend zu verbannen, wo er hingehört.

\section{Kontrollierte Kosten und hohe Qualität können einhergehen}

Es drängt sich die Frage auf: Was ist uns die Gesundheit wert? Sehr viel - lautet die Antwort des Betroffenen, sehr viel - so muss auch die Antwort unserer Gesellschaft lauten! Sehr viel, weil wir wegen der ausgezeichneten Leistungen Gewissheit haben, dass unsere Eltern im Krankheits- und Pflegefall gut versorgt sind und wir unsere Arbeit bzw. unsere Aktivitäten nicht aufgeben müssen. Sehr viel, weil gesunde Menschen arbeiten, einkaufen, konsumieren und einen Beitrag zum volkswirtschaftlichen Wachstum leisten. Sehr viel, weil die hohe Qualität unseres Gesundheitswesens und die stetige Weiterentwicklung der Medizin uns ermöglichen, die Gesundheitsleistungen - sei es in Form von Behandlungen, Arzneimitteln oder Medizinaltechnik - gewinnbringend ins Ausland zu exportieren.

Die Gesundheit steht Jahr für Jahr ganz weit oben im Sorgenbarometer der Schweizer Bürger. Wir von der FMH sind der Meinung, dass kontrollierte Kosten und hohe Qualität einhergehen können; mit einem ganzheitlichen Ansatz und einem Beitrag aller Beteiligten. Trotzdem müssen wir uns fragen: Was ist uns die Gesundheit wert? Oder anders formuliert: Wollen wir lieber gesunde Menschen oder ein grösseres Strassennetz?

Für mich, für alle Ärzte ist die Antwort klar! Wir wollen heilen. Wir wollen gesunde Frauen, Männer und Kinder! Es ist höchste Zeit, dass sich auch die Politik der Bedeutung und des Werts unseres Gesundheitswesens als Gesamtes bewusst wird.

Dr. med. Jacques de Haller, Präsident der FMH

\footnotetext{
* Auszug eines Referats, gehalten am 16. Juni 2010 im Rahmen des traditionellen FMH-Sessionsanlasses mit den Bundesparlamentariern, mit einem Beitrag von Doris Leuthard zum Thema «Volkswirtschaftliche Bedeutung des Gesundheitswesens». Einen Bericht zum Anlass sowie ein Interview mit Reiner Eichenberger, Professor für Finanzwirtschaft an der Universität Freiburg, lesen Sie in der Schweizerischen Ärztezeitung 26/27, welche am 30. Juni 2010 erscheint.
} 